\title{
Influencia de Algunos Parámetros sobre el Ciclo de un Motor de Encendido por Compresión
}

\author{
Carlos G. Villamar, Simón J. Fygueroa y María J. Martín \\ Universidad de los Andes, Facultad de Ingeniería, Escuela de Ingeniería Mecánica, \\ Departamento de Ciencias Térmicas, GRUMOTE, Núcleo Pedro Rincón Gutiérrez, Edif. B. \\ Ofic. 2E24, Mérida, Edo. Mérida-Venezuela (e-mail: villamar@ula.ve)
}

\begin{abstract}
Resumen
Se presentan una serie de estudios paramétricos sobre el ciclo termodinámico del motor de encendido por compresión empleando para ello un programa de computación. El programa permite estudiar el ciclo considerando la variación de la composición y propiedades del fluido de trabajo debidas a la presión, temperatura y riqueza de la mezcla. Se han efectuado estudios sobre la influencia de los principales índices del motor sobre la presión y temperatura promedio de los gases presentes en el cilindro. Entre estos se ha considerado, el ángulo de inicio, el adelanto y duración del proceso de combustión, la riqueza y la recirculación de gases. Se concluye que si aumenta la duración de la combustión se debe incrementar la riqueza para mantener la potencia y que la recirculación de gases produce una reducción de las emisiones de óxidos nitrosos.
\end{abstract}

\section{Effect of Some Parameters on the Performance of a Compression Ignition Engine}

\begin{abstract}
Some parametric studies on the thermodynamic cycle of a compression ignition engine by using a computer program that allows to analyze the cycle considering variations of working fluid composition and fluid properties due to pressure, temperature and fuel/air ratio. Studies on the influence of main indexes of the engine on the average pressure and temperature of the gases in the cylinder were done. Among these, the starting angle, the ignition time and duration of the combustion, the equivalence ratio, and gas recirculation, were considered. It is concluded that an increase in combustion duration implies a richness increase to maintain the power, and that nitrogen oxide emissions are reduced by gas recirculation.
\end{abstract}

Key words: engine, compression ignition, combustion, thermodynamic cycle, modeling 


\section{INTRODUCCIÓN}

Debido a la gran demanda y al incremento de los costos del combustible se hace necesario el desarrollo de técnicas que permitan hacer más eficientes los procesos energéticos. La comprobación de estas técnicas consume gran cantidad de tiempo y dinero. Una forma de reducir los costos que esto implica, es el empleo del modelado que mediante el uso de modelos matemáticos, en el caso de los motores térmicos, permite predecir su comportamiento para diferentes condiciones de funcionamiento. El proceso que más influencia tiene sobre el trabajo producido por un motor es la combustión, que es un proceso muy complejo que depende de una gran cantidad de variables. Para estudiar este fenómeno se han propuesto diversos modelos (Barba et al., 2000; Arsie et al., 2004, Arsie et al., 2006), que permiten predecir desde la evaporación del combustible, hasta la cantidad de energía liberada durante la combustión. Entre más preciso sea un modelo mayor será el tiempo de computo empleado ya que se verán involucradas mayor cantidad de variables.

En el presente trabajo se presentan los resultados de una serie de estudios paramétricos realizados con el modelo matemático propuesto por Villamar et al. (2007). El modelo tiene en cuenta el retardo de encendido, la autoencendido del combustible, la transferencia de calor a través de las paredes, el soplado (blow by), la presencia de gases residuales, la variación de la composición y propiedades del fluido de trabajo a lo largo del ciclo, la recirculación de gases residuales para controlar las emisiones de óxidos nitrosos y el avance y retardo de cierre de las válvulas de admisión y escape lo cual permite estudiar con adecuada fidelidad el comportamiento de los principales índices del ciclo teórico de los MEC de cuatro tiempos, durante el proceso de combustión

\section{METODOLOGÍA}

Se emplea el modelo termodinámico propuesto por Villamar et al. (2007) obtenido al aplicar las ecuaciones de la Primera Ley de la Termodinámica, continuidad y gases perfectos al volumen de control del cilindro.

$$
\begin{aligned}
& \frac{d p}{d t}=p\left[-\frac{\frac{d V}{d t}}{V}+\frac{\frac{d m}{d t}}{m}+\left(\frac{d T}{d t}\right) \frac{1}{T}-\frac{R T}{p}\left(\frac{\partial \rho}{\partial \phi}\right) \frac{d \phi}{d t}\right] \\
& \frac{d T}{d t}=\frac{B-\left(\frac{\partial u}{\partial p}\right) \frac{p}{D}\left[\left(\frac{\partial R}{\partial \phi}\right) \frac{d \phi}{d t} \frac{1}{R}+\frac{d m}{d t} \frac{1}{m}-\frac{d V}{d t} \frac{1}{V}\right]-\left(\frac{\partial u}{\partial \phi}\right) \frac{d \phi}{d t}}{\left(\frac{\partial u}{\partial T}\right)+\frac{C}{D} \frac{p}{T}\left(\frac{\partial u}{\partial p}\right)}
\end{aligned}
$$

donde:

$$
B=-R T \frac{\frac{d V}{d t}}{V}+\frac{1}{m}\left(\dot{Q}+\dot{m}_{e} h_{e}-\dot{m}_{s} h_{s}-\left(u \frac{d m}{d t}\right)_{v c}\right) ; \quad D=1-\frac{p}{R}\left(\frac{\partial R}{\partial p}\right) ; \quad C=1+\frac{T}{R}\left(\frac{\partial R}{\partial T}\right)
$$

$\mathrm{p}$ : presión dentro del cilindro; T: temperatura dentro del cilindro; V: volumen considerado; m: masa contenida en el volumen de control; $\rho$ : densidad de la mezclä; $\phi$ : riqueza de la mezcla; R: constante del fluido de trabajo; t: tiempo; u: energía interna específica; $\mathrm{Q}$ : flujo de calor y los subíndices; e, s, c, vc, se refieren a entrada, salida, combustible y volumen de control respectivamente.

Los términos $\frac{\partial u}{\partial T}, \frac{\partial u}{\partial p}, \frac{\partial R}{\partial T}$ y $\frac{\partial R}{\partial p}$ se determinan mediante las rutinas FARG y ECP (Ferguson y Kirkpatrick, 2001), mientras que los términos $\frac{\partial R}{\partial \phi}, \frac{\partial u}{\partial \phi}$ se calculan empleado la rutina PER (Olikara y Borman, 1975). El volumen instantáneo y su derivada se determina mediante las expresiones propuestas por Heywood (1988) y Van Wylen et al. (2003), mientras que el flujo másico que ingresa y sale del cilindro se hallan a partir de las expresiones propuestas por Zweiri et al. (2001), para determinar teóricamente el perfil de levantamiento de la válvula se emplea el modelo propuesto por Assanis (1990), se usa el modelo de soplado propuesto por Ferguson y Kirkpatrick (2001) para calcular la masa perdida a través de las zonas no herméticas del pistón. La cantidad de energía liberada duran- 
te la combustión se halla a partir de la masa de combustible quemado que se expresa en función de dos términos, la quemada durante la fase premezclada y la quemada durante la fase difusiva (Assanis et al., 2003). Para tener en cuenta el retardo de encendido el modelo emplea la expresión propuesta por Watson et al. (1980). Para calcular el coeficiente promedio de transferencia de calor por convección se emplea la correlación propuesta por Hohenberg (1979). La composición de los productos de combustión y sus propiedades se determinan empleando un modelo CHON de diez especies químicas. La concentración de óxidos de nitrógeno presente en los gases de escape se determina a partir de la teoría cinética química, para ello se utiliza el mecanismo de Zeldovich (Egnell, 2000). El sistema formado por las Ecs. (1) y (2) se resuelve empleando los modelos descritos y la rutina DVERK (Ferguson y Kirkpatrick, 2001), que permite resolver sistemas de ecuaciones diferenciales ordinarias.

Para llevar a cabo los estudios paramétricos se utilizaron los datos geométricos de un motor CFR que se muestran en la Tabla 1 , y se ejecutó repetidamente el programa correspondiente manteniendo invariable algunos parámetros y permitiendo la variación de otros como se ilustra en la Tabla 2.

Tabla 1. Dimensiones del motor CFR y características del lugar de ensayo.

\begin{tabular}{|c|c|c|c|c|c|c|c|c|c|c|}
\hline $\begin{array}{c}\mathrm{D}_{\mathrm{c}} \\
(\mathrm{mm})\end{array}$ & $\begin{array}{c}\mathrm{c} \\
(\mathrm{mm})\end{array}$ & $\begin{array}{c}\mathrm{d}_{V_{a}} \\
(\mathrm{~mm})\end{array}$ & $\begin{array}{c}\mathrm{d}_{\mathrm{Ve}} \\
(\mathrm{mm})\end{array}$ & $\begin{array}{c}\mathrm{L}_{V \max } \\
(\mathrm{mm})\end{array}$ & $\begin{array}{c}\mathrm{L}_{\mathrm{Vmax}} \\
(\mathrm{mm})\end{array}$ & $\begin{array}{c}\mathrm{p}_{\mathrm{atm}} \\
(\mathrm{kPa})\end{array}$ & $\begin{array}{c}\mathrm{T}_{\mathrm{amb}} \\
(\mathrm{K})\end{array}$ & $\begin{array}{c}\mathrm{T}_{\mathrm{p}} \\
(\mathrm{K})\end{array}$ & $\begin{array}{c}\mathrm{AAA} \\
\left({ }^{\circ}\right)\end{array}$ & $\begin{array}{c}\mathrm{RCE} \\
\left({ }^{\circ}\right)\end{array}$ \\
\hline 82.55 & 114.3 & 31.75 & 31.75 & 7.86 & 8.9 & 100 & 300 & 500 & 0 & 0 \\
\hline
\end{tabular}

Donde: $D_{c}$ : Diámetro del cilindro; c: carrera del pistón; $d_{v a} y d_{v e}$ : diámetro de las válvulas de admisión

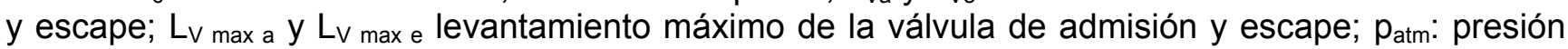
atmosférica; $\mathrm{T}_{\mathrm{amb}}$ : temperatura ambiente; $\mathrm{T}_{\mathrm{p}}$ : temperatura de la pared del cilindro; $\mathrm{AAA}$ : ángulo de avance de apertura de la válvula de admisión y RCE: retardo del cierre de la válvula de escape.

Tabla 2. Parámetros fijos y variables para cada estudio.

\begin{tabular}{|c|c|c|}
\hline Tipo de estudio paramétrico & Parámetros fijos & $\begin{array}{l}\text { Parámetros } \\
\text { variables }\end{array}$ \\
\hline 1. Influencia del inicio de la inyección del combustible & $\begin{array}{c}\phi=0.85 ; r_{c}=18 ; n=2000 \\
\Delta \varphi=50^{\circ}\end{array}$ & $\varphi_{i \text { comb }}$ \\
\hline $\begin{array}{l}\text { 2. Influencia de la duración del proceso de combus- } \\
\text { tión }\end{array}$ & $\begin{array}{c}\phi=0.85 ; r_{c}=18 ; n=2000 \\
\varphi_{i \text { comb }}=345^{\circ}\end{array}$ & $\Delta \varphi$ \\
\hline $\begin{array}{l}\text { 3. Influencia del avance de inyección al variar el ré- } \\
\text { gimen de giro }\end{array}$ & $\phi=0.85 ; r_{c}=18 ; \Delta \varphi=45^{\circ}$ & $\mathrm{n} ; \varphi_{\mathrm{i} \text { comb }}$ \\
\hline $\begin{array}{l}\text { 4. Influencia de la recirculación de gases residuales } \\
(E G R) \text { sobre la formación de óxidos nitrosos }\end{array}$ & $\begin{array}{l}\phi=0.85 ; r_{c}=18 ; n=2000 \\
\varphi_{i ~ c o m b}=345^{\circ} ; \varphi_{\text {f comb }}=390\end{array}$ & $\% E G R$ \\
\hline
\end{tabular}

Donde: $n$ : frecuencia de giro del motor; $r_{c}$ : relación de compresión; $\varphi_{\mathrm{i}}$ comb $\mathrm{y} \varphi_{\mathrm{f} \text { comb }}$ : ángulo de inicio y finalización de la combustión; $\Delta \varphi$ : duración del proceso de combustión.

\section{RESULTADOS Y ANALISIS}

\section{Influencia del inicio de la inyección de combustible}

Las Fig. 1 y 2 muestran como varía la temperatura y presión dentro del cilindro. Puede observarse que al avanzar el inicio del proceso de combustión se alcanzan mayores presiones, la mayor parte durante la carrera de compresión, lo que trae como consecuencia mayor consumo de trabajo y menor eficiencia del ciclo. Al retardarse el inicio del proceso de combustión se reduce la presión máxima. Al avanzar el inicio del proceso de combustión de manera que se produzca alrededor del PMS se obtienen presiones máximas intermedias que producirán un trabajo de expansión relativamente mayor que el consumido durante la compresión.

\section{Influencia de la duración del proceso de combustión}

En la Fig. 3 se observa que a menor duración del proceso de combustión mayor será la presión Información Tecnológica Vol. - 20 N¹ - 2009 
máxima alcanzada por liberarse la energía en un lapso menor, como se puede comprobar con la pendiente de las curvas de la Fig. 4. en la que se nota que para los casos considerados los valores máximos de masa quemada no cambian; esto se debe a que se mantiene constante la riqueza y puesto que la eficiencia volumétrica prácticamente no cambia, entonces la masa quemada dentro del cilindro será la misma para todos los casos.

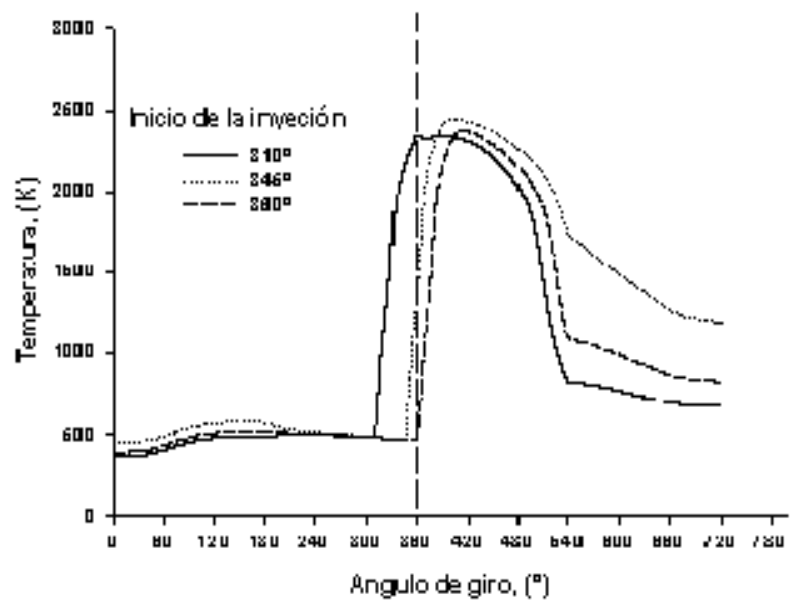

Fig. 1: Variación de temperatura en el cilindro

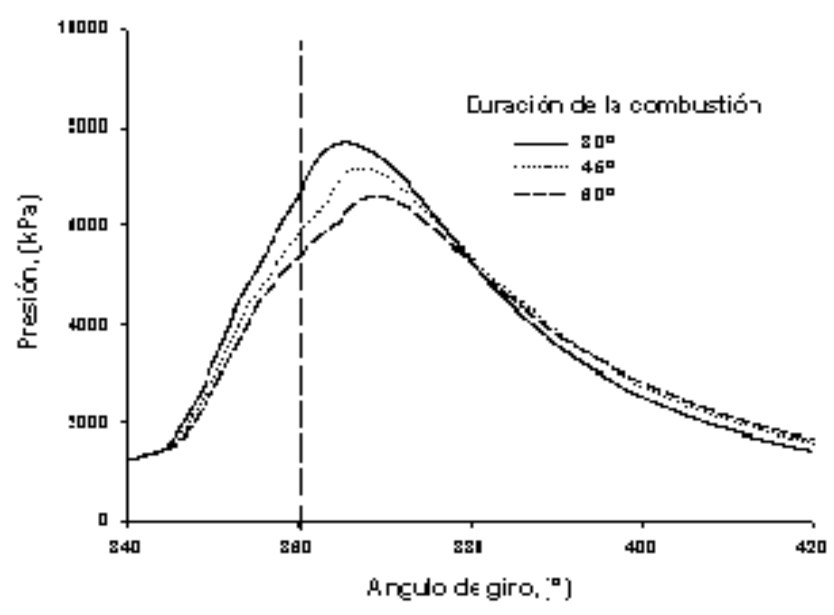

Fig. 3: Variación de presión en el cilindro

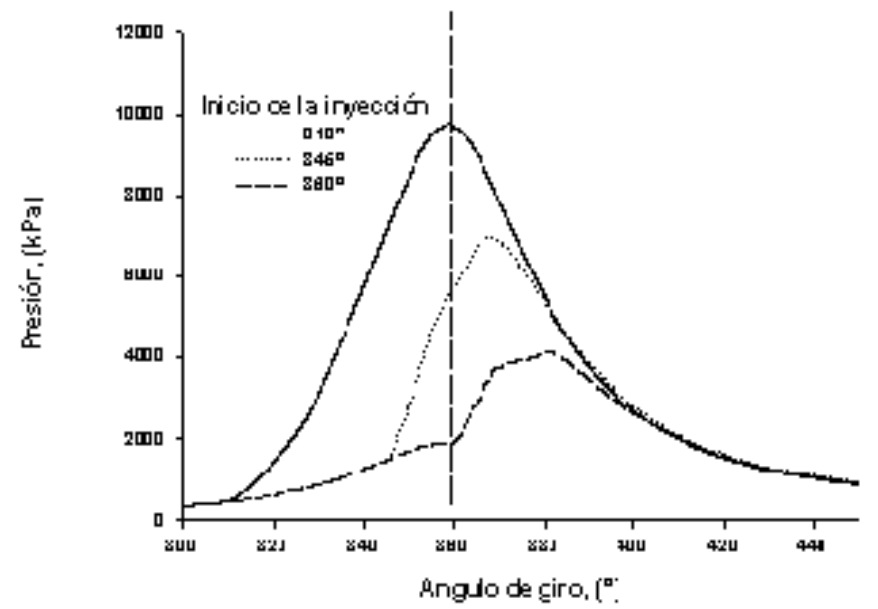

Fig. 2: Variación de la presión en el cilindro

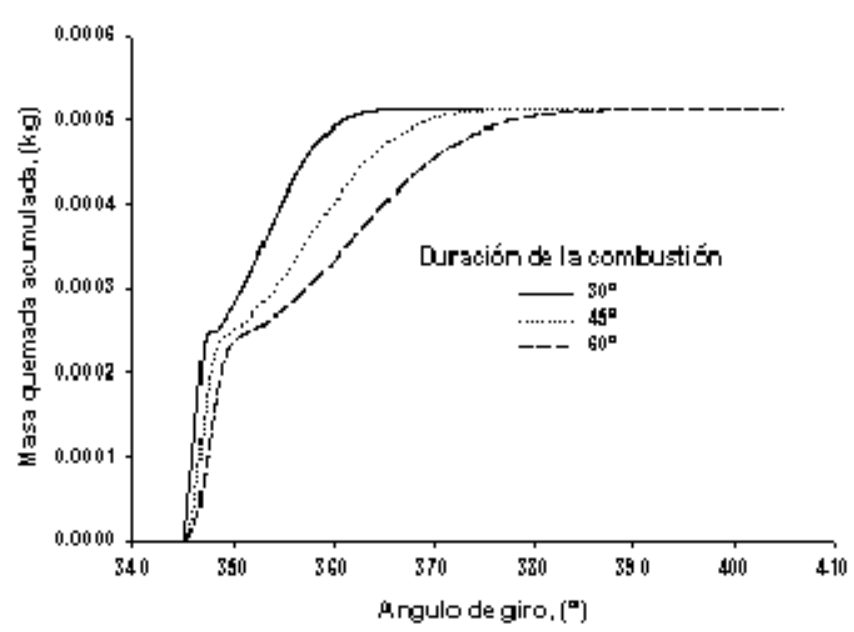

Fig. 4: Masa quemada acumulada

Influencia del avance de inyección al variar el régimen de giro

La Fig. 5 muestra el diagrama de indicador para diversos regímenes de giro del motor con adelanto del inicio del proceso de combustión. De manera que la presión máxima para los casos estudiados ocurra alrededor del PMS y su valor máximo se mantenga aproximadamente constante. De esta manera se puede encontrar una primera aproximación del ángulo óptimo de inicio de la combustión para cada frecuencia, que es lo que se busca en un motor real de manera que se obtengan la máxima eficiencia y potencia en un amplio rango de regímenes de giro (Heywood, 1988).

Influencia de la recirculación de gases residuales (EGR) sobre la formación de óxidos nitrosos

Una forma de reducir las emisiones de óxidos nitrosos es recirculando gases residuales (Egnell, 2000; Sangjin et al., 2004). En la Fig. 6 se puede apreciar que la presión máxima del ciclo se obtiene cuando no hay recirculación de gases. El uso de EGR, en sustitución de cierta cantidad del aire que ingresa al cilindro con gases de escape, afecta el proceso de combustión, lo que se manifiesta con una reducción de la presión y temperatura dentro del cilindro del motor. De esta manera se consigue una disminución de las emisiones de óxidos nitrosos, como puede observarse en la Fig. 7 donde la concentración máxima de NO se obtiene cuando no se emplea EGR. Aunque la concentración de $\mathrm{NO}$ es menor a mayor porcentaje de EGR, la potencia producida por el motor se reduce ya que dis- 
minuye la cantidad de aire que ingresa al cilindro, lo que a su vez reduce la cantidad de calor liberado durante el proceso de combustión. En la Fig. 8 se presenta a manera de comparación, la variación de la concentración de NO obtenida empleando la teoría del Equilibrio Químico y el modelo de Zeldovich. Se puede apreciar que ésta prácticamente no presenta variación durante la expansión pero si durante el escape

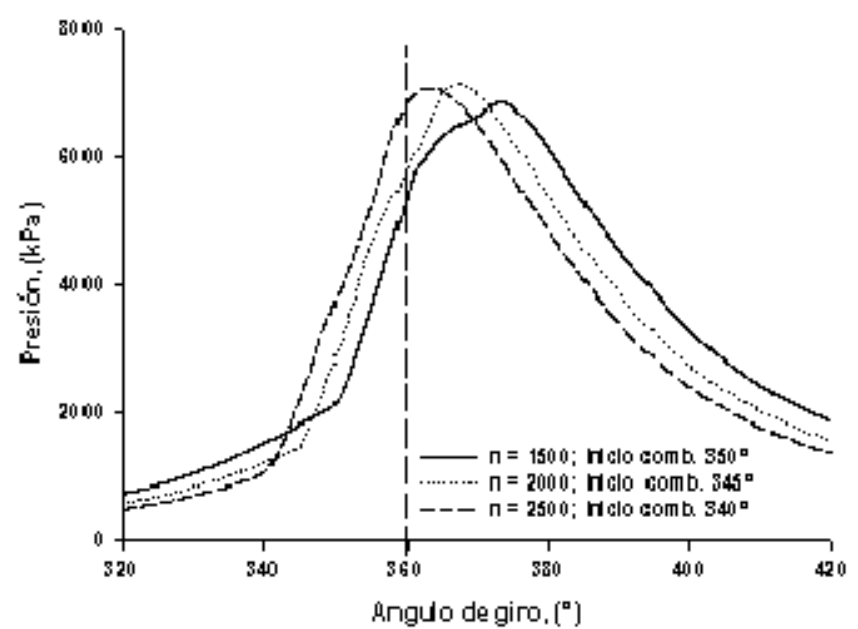

Fig. 5: Variación de la presión en el cilindro

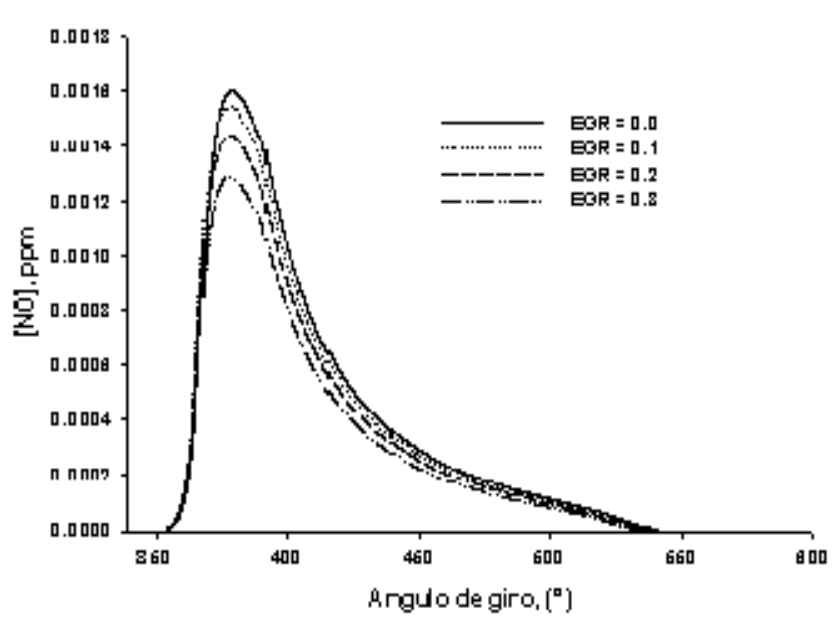

Fig. 7: Variación de la concentración de NO para diferentes porcentajes de EGR

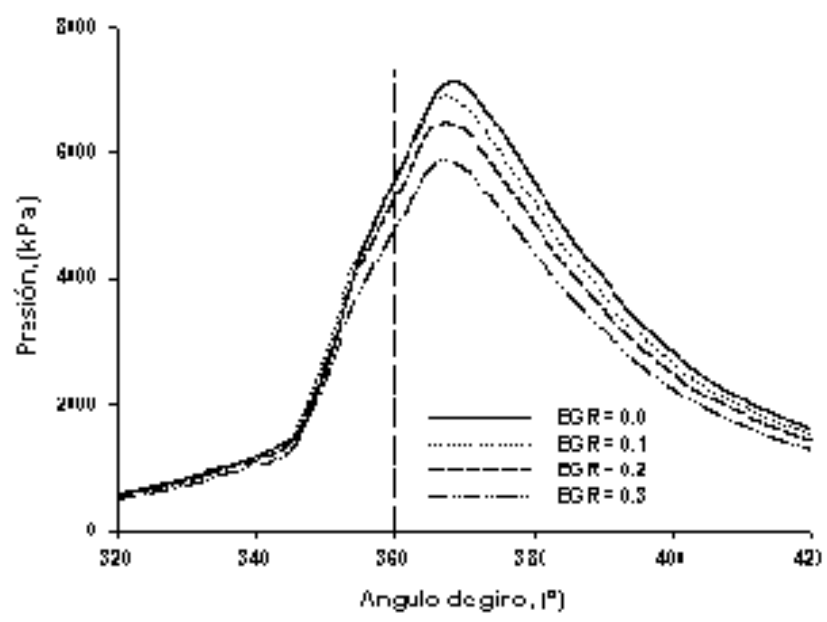

Fig. 6: Variación de la presión en el cilindro empleando varios porcentajes EGR

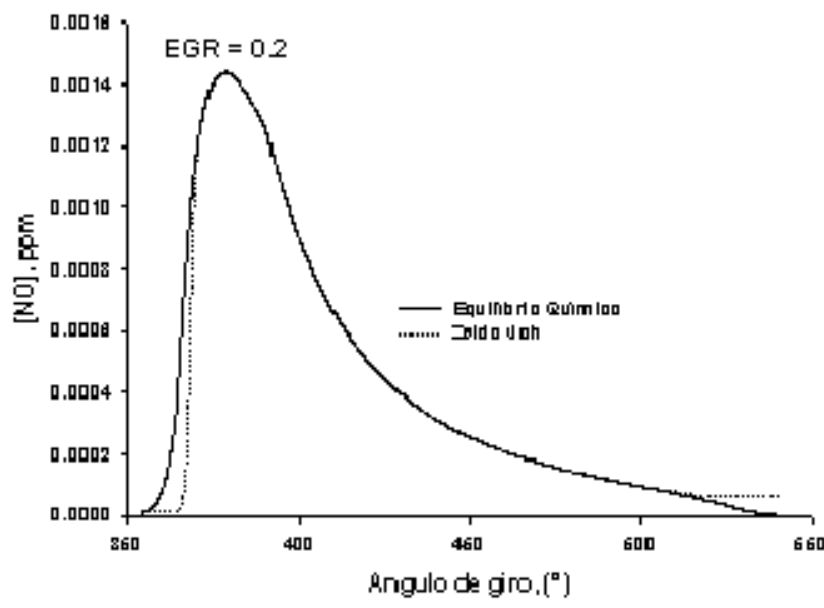

Fig. 8: Variación de concentración de NO según la teoría de equilibrio químico y el modelo de Zeldovich

\section{CONCLUSIONES}

De los estudios paramétricos realizados se obtienen las siguientes conclusiones:

Para obtener mayor trabajo del ciclo se deben seleccionar los parámetros de manera que el proceso de combustión se produzca en el menor tiempo posible siempre y cuando las presiones máximas estén dentro del rango tolerable desde el punto de vista de la resistencia de los materiales.

Al aumentar la duración del proceso de combustión se debe aumentar la riqueza con la finalidad de compensar la caída de presión dentro del cilindro para de esta forma mantener la potencia producida por el motor.

Al reemplazar una parte del aire por gases residuales se reduce la temperatura en el cilindro lo que trae como consecuencia una disminución de las emisiones de óxidos nitrosos; de esta manera se 
demuestra la utilidad del empleo de dicha técnica para controlar las concentraciones de NO presentes en los gases de escape.

\section{REFERENCIAS}

Arsie I. y otros siete autores; Development and Identification of Phenomenological Models for Combustion and Emissions of Common-Rail Multi-Jet Diesel Engines, SAE Paper: 2004-01-1877 (2004).

Arsie I. y otros siete autores; Multi-Zone Predictive Modeling of Common-Rail Multi-Injection Diesel Engines, SAE Paper: 2006-01-1384 (2006).

Assanis, D.; Valve Optimization in a Spark Ignition Engine, Journal of Engineering for Gas Turbines and Power: 112, 341-347 (1990).

Assanis D., S. Zoran y J. Timothy; The Impact of Exhaust Gas Recirculation on Performance and Emission of a Heavy Duty Diesel Engine, SAE Paper: 2003-01-1068 (2003).

Barba C., C. Burkhardt, K. Boulouchos, M. Bargende; A Phenomenological Combustion Model for Heat Release Rate Prediction in High-Speed DI Diesel Engines Whit Common Rail Injection, SAE Paper: 2000-01-2933 (2000).

Egnell, R.; The Influence of EGR on Heat Release Rate and NO Formation in a DI Diesel Engine, SAE Paper: 2000-01-1807 (2000).

Ferguson C. y A. Kirkpatrick; Internal Combustion Engines, Applied Thermosciences, pp. 384, John Wiley and Son (2001).

Heywood J.; Internal Combustion Engine Fundamentals, pp 930, Mc. Graw Hill, New York (1988).

Hohenberg, G.; Advanced Approaches for Heat Transfer Calculations, SAE Paper: 790825 (1979).

Olikara, C. y G. Borman; A Computer Program for Calculating Properties of Equilibrium Combustion Products with Some Applications to I.C. Engines: SAE Paper 750468 (1975).

Sangjin, H., D. Assanis, M. Wooldridge y H. Im; Modeling of Diesel Combustion and NO Emissions Based on a Modified Eddy Dissipation Concept, SAE Paper: 04P-273 (2004).

Van Wylen, R. Sonntag, C. Borgnake; Fundamentos de Termodinámica, pp. 891. Limusa Wyley (2003).

Villamar C., S. Fygueroa y J. Araque; Modelo Termodinámico de los Motores de Encendido por Compresión. $8^{\circ}$ Congreso Iberoamericano de Ingeniería Mecánica, Cusco, Perú (2007).

Watson, N. A. Pilley y M. Marzouk; Combustion Correlation for Diesel Engine Simulation: SAE Paper 800029 (1980).

Zweiri, Y., J. Whidborne y L. Senevirante; Detailed Analytical Model of a Single-Cylinder Diesel Engine in the Crank Angle Domain, Proc. Instn. Mech. Engrs.; Part D, 215, 1197-1216 (2001). 